\title{
L'ENDOCRINOLOGIE COMBINATOIRE
}

\section{Jacques Hanoune}

\author{
ADRESSE \\ I. Hanoune: direcleur de recherche i l'mserm. \\ Inserm U. 99, Hôpital Henri-Mondor, 51, \\ arenue du Narechal-de-l attre-de-Tassigns; \\ 94010 Créteil, France.

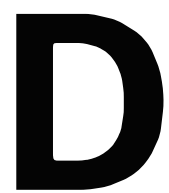

ans l'histoire de l'endocrinologie qui, au sens large, peut être considérée comme la discipline des régulations, un concept simple, clair et unifiant a de temps en temps émergé. (itons par exemple la décourerte de l'insuline, la notion de récepteur hormonal, ou bien le sustème de l'AMPc. A l'heure actuelle, c'est plutôt à un phénomène inverse que nous assistons. La littérature foisonne de facteurs régulateurs sans qu’il soit toujours possible d'apprécier leur impact physiologique réel. L.e temps des modèles simples est-il passé? L'endocrinologie est-clle devenue combinatoire "'e numéro de médecine/sciences, qui regroupe un certain nombre d'articles dans le domaine de l'endocrinologie, encourage à poser ces questions.

Prenons comme exemple l'action de l'adrénaline sur le taux d'AMPc. Longtemps après l'avoir découvert en 1958 , E.W. Sutherland présentait encore un modèle très simple [1]: une "boîte" membranaire unique recomnaissant le signal hormonal et svinthétisant l'AMPc, qu'une phosphodiestérase intracellulaire se chargeait de détruire. Il n’apparaissait pas du tout que l'adénvilyl cyclase (enzvime que la simplicité des temps appelait encore adényl cyclase) devait être distinguée du récepteur. Dans un autre modèle de l'époque, on attribuait même l'effet activateur de l'adrénaline à l'existence d'un complexe avec l'ATP, dans lequel l'hormone était supposée aider, comme un cofacteur, au positionnement correct des phosphates de l'ATP dans le site catalytique de l'enzrme grâce aux deux hydroxvles de son novau catéchol [2]. Il a fallu attendre les amnées 197() pour que récepteur et enzyme soient conceptuellement distingués, la première démonstration biochimique devant attendre la purification du récepteur $\beta$-adrénergique [3]. Puis la découverte des protéines (; est venue ajouter un degré de complexité supplémentaire.

A l'heure actuelle, le srstème de l'AMPc apparaît tout autre, car il implique plusieurs dizaines d'entités protéiques différentes. On comnait au moins une dizaine de récepteurs adrénergiques qui interagissent positivement $\left(\beta_{1}, \beta_{3}, \beta_{3}\right)$, négativement $\left(\alpha_{2}\right)$ ou indirectement par l'intermédiaire du (á ${ }^{2+}$ ou de la protéine kinase (: $\left(\alpha_{1}\right)$ avec l'adénvilyl cyclase. Un article de D). Strosberg dans le dernier numéro de médecine/sriences $\left(n^{\circ} 11, v o l .9, p .1228\right)$ fait d'ailleurs le point sur le récepteur $\beta_{3}$ adrénergique. L'action de l'adrénaline sur un tissu donné ra donc dépendre, en premier lieu, de la stoechiométrie respective des différents types de récepteurs présents dans un tissu domné, et de leur affinité pour l'hormone circulante. ('est ainsi que l'adrénaline pourra être lipolstique ou antilipolytique, en fonction de la topographie ou du type du tissu adipeux, du sexe, de l'espèce animale, etc. A l'inverse, 
l'adrénaline est glycogénolytique dans toutes les espèces, mais emprunte la voie de l'AMPc chez le chien ou le lapin, (récepteur $\beta_{2}$ ) et celle du calcium (récepteur $\alpha_{1}$ ) chez le rat. Dans le même esprit, il vient d'être montré que l'augmentation de l'AMPc des cardiomyocytes sous l'effet du glucagon était due à une activation de l'adénylyl cyclase chez le rat, mais à une inhibition de la phosphodiestérase chez le cobaye ou la grenouille [4]. Une même hormone peut donc emprunter des systèmes différents de transduction du signal.

I.es protéines $G$ sont encore plus complexes puisque l'on dénombre à l'heure actuelle 21 sous-unités $\alpha_{1}$ (produits de 17 gènes différents), 4 sous-unités $\beta$ et 7 sous-unités $\gamma[5]$. Les nombres ne sont pas définitifs. Dans quelques systèmes privilégiés oì cela a été étudié, on a pu démontrer que les sous-unités $\beta$ ou $\gamma$ n'étaient pas équivalentes, mais que chaque récepteur pourrait, en quelque sorte, être précouplé à un système de sous-unités différent. (;. Schultz vient ainsi de montrer, dans les cellules (iH3, que trois effecteurs inhibaient le même canal calcique sensible à la toxine de Bordetella pertussis, la somatostatine par le complexe $\alpha_{102}-\beta_{1}-\gamma_{3}$, l'acétvlcholine par le complexe $\alpha_{11}-\beta_{2}-\gamma_{4}$, et la galanine par le complexe $\alpha_{01}-\beta_{2}-\gamma_{2}$. Ainsi, chaque effecteur a son propre code composé d'une combinaison spécifique de sous-unités.

Enfin, à l'étage suivant, l'adénylyl cyclase présente au moins huit types différents [6], chacun étant le produit d'un gène propre (Haber et al., soumis pour publication). Ces différents types sont tous activés par la forskoline ou la sous-unité $\alpha_{s}$, mais le complexe calcium-calmoduline active les types I et III; le calcium inhibe les types V et VI; la phosphorylation sous l'effet de la protéine kinase $C$, active le type II. I.e phénomène le plus intéressant est le rôle ambigu des sous-unités $\beta \gamma$ qui activent les types II et IV et inhibent directement le type I [7]. Cies résultats inattendus pourraient rendre compte de constatations surprenan- l'adénylyl cyclase par des hormones qui activent non pas la sous-unité $\alpha_{s}$, mais les sous-unités $\alpha_{0}$ ou $\alpha_{i}$. Dans ces cas, la stimulation est indirecte, liée à une augmentation du $\mathrm{Ca}^{2+}$ intracellulaire [8] ou à une libération de $\beta \gamma$ à partir de leur complexe avec $\alpha_{0}$ ou $\alpha_{i}$ [9]. Dès lors, la spécificité de la réponse physiologique d'un tissu à une hormone n'apparaît plus étroitement liée au seul récepteur, mais aussi bien aux différentes cascades possibles d'amplification ultérieure et à leur interaction, et en particulier la nature et la stœechiométrie, propres à chaque cellule, des protéines $\mathrm{G}$ et des systèmes effecteurs. C.es mêmes notions s'appliquent pour la variation de la sensibilité d'une cellule donnée à une hormone au cours du développement. De plus, l'AMPc, une fois formée, est dégradée par la phosphodiestérase dont il existe au moins 25 formes, classées en 5 familles comme le décrivent $B$. Muller et al. dans le présent numéro (p. 1335), chaque famille présentant une régulation propre. Dès lors, comme le dit C. de Rouffignac (p. 1367 de ce numéro), la régulation plurihormonale d'une fonction complexe, comme la fonction rénale par exemple, ne peut plus s'expliquer par des effets simples et univoques de quelques facteurs hormonaux, mais résulte forcément d'une combinatoire d'événements.

L'AMPc, outre son action classique sur l'état de phosphorylation d'une large population d'enzymes par la protéine kinase $\mathrm{A}$, agit aussi au niveau du génome. Là, il met en jeu, comme le dit Habener, une "corne d'abondance" de facteurs transcriptionnels [10]. Le facteur le plus connu, CREB, voit son activité augmentée par phosphorylation sous l'effet de la PKA. Il recouvre en fait toute une famille de protéines dont certains variants, comme CREM, agissent comme inhibiteur dominant négatif de CREB $\left(\mathrm{m} / \mathrm{s} n^{\circ} 5\right.$, vol. 7, p. 506). Le gène (REM lui-même a fait l'objet de nombreux travaux et se trouve impliqué dans le contrôle de la spermatogenèse et du rythme circadien (m/s $n^{\circ} 11$, vol. 9, p. 1253). Ia propriété d'hétérodimérisation entre facteurs transcriptionnels avec ses lois propres de légitimité fournit un répertoire riche de possibilités. D'ailleurs, la frontière de la notion de légitimité doit être reculée tous les jours. Ne voit-on pas la protéine CREB, activable par la protéine kinase A et l'AMPc former un hétérodimère avec la protéine Jun activable par la protéine kinase (C et les esters de phorbol?

On retrouve ainsi au niveau génique la même complexité combinatoire des mécanismes de régulation que celle rencontrée au niveau membranaire. L'étude des grandes voies de la glycolyse et de la gluconéogenèse a conduit depuis 30 ans au concept simple que les enzymes clés impliquées dans ces voies pouvaient être classées en deux groupes, l'un responsable de la voie de synthèse du glucose à partir des composés à trois atomes de carbone, induit par les glucocorticoïdes, l'AMPc et inhibé par l'insuline, l'autre responsable de la voie glycolytique, induit par l'insuline et réprimé par l'AMPc. (On a même pu émettre l'hypothèse qu'un master gene ou master stwitch pouvait commander chaque famille. L'étude des promoteurs de chacune de ces enzymes conduit à des conclusions beaucoup plus désabusées: il n'existe pas de "promoteur consensus" pour chaque voie métabolique. Chaque promoteur possède son complément propre de GRE (glucose response element), (CRE (cyclic AMP response element), etc., qu'il possède ou non une TATA box, et semble dépendre d'une combinaison originale de facteurs régulateurs interagissant directement ou indirectement avec l'ADN. A ce jour, on n'est pas encore en mesure d'identifier un IRE (insulin response elememt) universel et l'interaction positive ou négative entre les différents éléments de régulation apparaît d'autant plus complexe que les facteurs spécifiques des tissus se surajoutent aux facteurs transcriptionnels sensibles aux hormones. F. Jacob [11] a proposé pour ces complexes protéiques variables la terminologie d'agrégulat (contraction d'agrégat et de régulation), chaque système de régulation génique sem- 
blant avoir trouvé une combinaison propre de facteurs de contrôle, combinaison fixée par l'évolution dans un processus que le même auteur a joliment résumé sous le nom de "bricolage". Au niveau des régulations membranaires, les interactions imprévisibles entre les différentes voies de signalisation sont résumées par le vocable moins heureux de cross talk[12]. Dans les deux cas, comment détecter ce qui est déterminant, redondant ou même accessoire? On peut espérer qu'une analyse toujours plus détaillée fera apparaitre des invariants mettant sur la piste de nouveaux modèles. I a voie de signalisation de ras en est un exemple éclatant [13] (voir aussi $\mathrm{m} / \mathrm{s} n^{\circ} 5$, vol. $\left.8, p .471\right)$. L'utilisation de sristèmes "antisens", sur des cellules en culture ou chez des animaux transgéniques, la mise en ceuvre, lourde, de knock-out de gènes, pourront permettre de progresser dans l'élucidation des hiérarchies de contrôle. Mais n'oublions pas que les modèles pathologiques, che' l'homme ou l'animal, ont le mérite de souligner brutalement les voies majeures de régulation. (On peut noter un exemple récent, celui de l'adénome hrperfonctionnel ou toxique de la thiroïde. Sur le plan biochimique, il apparaît lié à un hyperfonctionnement du sustème adénvlate crclasique. On commaît maintenant les anomalies moléculaires qui peuvent l'expliquer dans certains cas : il s'agit d'une activation permanente, par mutation ponctuelle, de la protéine Gs $\alpha$ [14] ou (ii $\alpha$ [15] ou encore du récepteur de la TSH, comme vient de le montrer brillament le groupe de G. Vassart ([16] et p. 1421 de (e numéro). Ces anomalies n'expliquent pas tous les cas cliniques, mais quelle meilleure démonstration du rôle majeur d'une voie de régulation?

\section{RÉFÉRENCES}

1. Sutherland Fill: (n) the biological role of crclic AMP. I Am Med Ass 197(): 214: $1281-8$.

2. Belleau B. Stereochemistry of adrenergic receptors: newer concepts on the molecular mechanism of action of catecholamines and antiadrenergic drugs at the receptor level. Ann . Vi Acaul Sci 1967\%: 139): 58()$-(6) 5$

3. V'auquelin (;, Gernet P, Hanounc J Strosberg 1). Isolation of adenvlate crclasefree, $\beta$ adrenergic receptor from turkey ervthrocste membranes by affinity chromatography. Pror Nall Arad Sci LSS 1977; 74 371()$-4$.

4. Brechler V', Paroine (;, Hanf R, (Barbaty, F, Fischmeister R, Pecker F. Inhibition by glucagon of the c(;.MP-inhibited low-Km (A.MPP phosphodiesterase in heart is mediated by a pertussis toxin-sensitive (-protein. I Biol chem 1992: 267: $1549(250) 1$.

5. Hepler JR, Gilman Ac;. (; proteins. Trends Biochem Sci 1992; i7: 383-7.

6. Mengar R. Molecular and functional diversits of mammalian (sis-stimulated

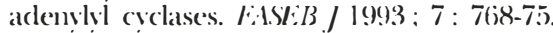

7. (lapham 1)E, Neer EJ. New roles for (; protein $\beta \gamma$ dimers in tansmembrane signalling. Nalure 19993: 365: 4(0)3-6.

8. (heckorich 1).M. (Grat R, Johnston 1), Sweatt J1). N-methrl-1)-aspartate receptor activation increase's c.A.MP levels and vollage gated $\left(\mathrm{a}^{++}\right.$chammel activity in area (i.Al of hippocampus. Proc Nall Acad Sci e.S 1991:88: $6467-71$.
9. Baugold J. Muscarinic receptor-mediated stimulation of adensivl crclase. Trends Physiol Sri 1992: 13: 339-40.

10. Habener JF. (velic A.MP response element binding proteins: a comucopia of transcription factors. Mol Findocinol 1990): 4: $1087-9.4$

11. Jacob) F. Du répresseur à l'agrégulat. (KR Acrid Sci Paris Sor III 1993: 316 : $547-9$.

12. Houslaw MI). "(irosstalk": a pirotal role for protein kinase (: in modulating relationships between signal transduction pathwass. Lidr / Biochem FlBBS 1991: 195: 927.

13. Fgan SE, Weinberg $R t$. The pathway to signal achievement. Nature 1993: 365 : $781-3$.

14. ()'Sullivan (: Banton (:.M. Stadden Sl., Brown (:I. I emone NR. Activating point mutations of the gse oncogene in human thwoid adenomas. . Iol Carrinogenesis 1991: $4: 345-9$.

15. Sclere F, Wilfing A, Schiferer A, Hermann .11, (irubeck-loebenstein, Freissmuth M. Stimulation of human throsicl growth ria the inhibitory guanine micleotide binding ((;) protein ( $\mathrm{ii}$ : constitutive expression of (j-protein a subunit ( $j_{i k t}$ in autonomous adenoma. Proc . Nall tead So ('S.1 1993: 90): 1609)-13.

16. ParmaJ, Dupres I., Van Sande.J. Cochaux P, (jerve (: Mockel], Dumomi). lassart (;. Somatic mutations in the thro tropin receptor gene cause hyperfunctioning throid adenomas. Nature 1993: 365 : (i.4.)-5).

\section{TIRÉS A PART}

